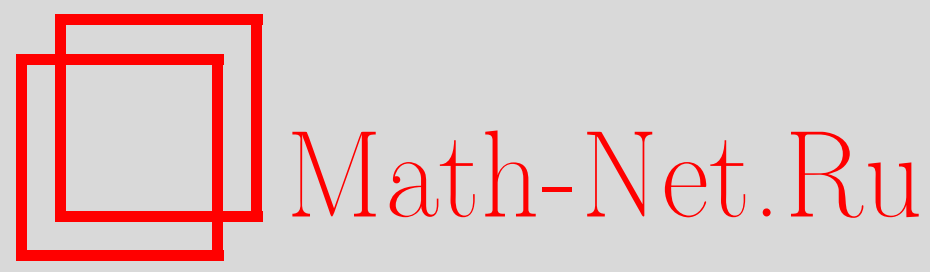

В. В. Ейко, А. А. Кондратюк, Об интегральных логарифмических средних произведений Бляшке, Матем. заметки, 1998, том 64, выпуск 2, 199-206

DOI: https://doi.org/10.4213/mzm1386

Использование Общероссийского математического портала Math-Net.Ru подразумевает, что вы прочитали и согласны с пользовательским соглашением http://www.mathnet.ru/rus/agreement

Параметры загрузки:

IP: 34.229 .108 .108

26 апреля 2023 г., $16: 16: 08$

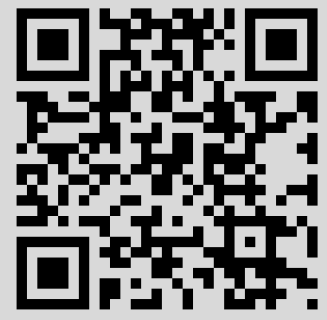




\title{
ОБ ИНТЕГРАЛЬНЫХ ЛОГАРИФМИЧЕСКИХ СРЕДНИХ ПРОИЗВЕДЕНИЙ БЛЯШКЕ
}

\author{
В.В. Ейко, А. А. Кондратюк
}

\begin{abstract}
С помощью метода рядов Фурье для мероморфных функций обобщаются результаты, характеризующие поведение интегральных логарифмических средних произведений Бляшке произвольного порядка.

Библиографиял: 7 названий.
\end{abstract}

Пусть $\left\{a_{n}\right\}$ - последовательность комплексных чисел, $0<\left|a_{n}\right|<1$, которые удовлетворяют условию Бляшке

$$
\sum_{n}\left(1-\left|a_{n}\right|\right)<\infty .
$$

Обозначим через $\mathscr{B}_{a}$ класс произведений Бляшке

$$
B(z)=\prod_{n} \frac{\bar{a}_{n}}{\left|a_{n}\right|} \frac{a_{n}-z}{1-\bar{a}_{n} z}
$$

для которых $a \leqslant\left|a_{n}\right|$, где $a>0$. Интегральные логарифмические средние произведения Бляшке порядка $q, 1 \leqslant q<\infty$, определим соотношением

$$
m_{q}(r, B)=\left(\frac{1}{2 \pi} \int_{-\pi}^{\pi}|\ln | B\left(r e^{i \theta}\right)||^{q} d \theta\right)^{1 / q}
$$

Пусть $n(t)=n(t, B)$ - количество членов последовательности $\left\{a_{n}\right\}$, которые удовлетворяют условию $a \leqslant\left|a_{n}\right| \leqslant t, n \in \mathbb{N}$.

Задача оценки $m_{2}(r, B)$ через $n(r, B)$ была поставлена А. Зигмундом. В 1969 году она была решена Г. Маклейном и Л. Рубелом [1]. Исследуем обший случай, когда $1 \leqslant q<+\infty$.

Некоторые результаты, характеризующие поведение интегральных логарифмических средних порядка $q, 1 \leqslant q<\infty$, произведений Бляшке и Цудзи-Джрбашяна были получены К. Линденом [2]-[4].

Используя метод рядов Фурье для мероморфных функций [1], [5], мы находим оценки (точные в определенном смысле) для $m_{q}(r, B)$ в терминах функции $n(r, B)$. Сначала сформулируем основные результаты настоящей статьи. Всюду далее $p$ и $q$ сопряжены, т.е. $1 / p+1 / q=1$. 
Tеорема 1. Пусть $B \in \mathscr{B}_{a}$. Тогда для кажсдого $q \in[1,+\infty)$ существует постоянная $A_{q}$ такая, что

$$
(1-r)^{1 / p} m_{q}(r, B) \leqslant A_{q} \int_{r}^{1} \frac{n(t)}{t} d t
$$

для всех $r, 0<r<1$.

СлЕДСТвИЕ (см. также [2]). Если $B \in \mathscr{B}_{a}, q \in[1,+\infty) u n(r)=O\left((1-r)^{-\alpha}\right)$, $0<\alpha<1$, mo $m_{q}(r, B)=O\left((1-r)^{1 / q-\alpha}\right), r \rightarrow 1$.

Теорема 2. Пусть нули произведения Бляике $B, B \in \mathscr{B}_{a}$, положительнь. Если существует $\beta, 0<\beta<1$, такое, что

$$
n(r)(1-r) \leqslant \beta \int_{r}^{1} n(t) d t, \quad 0<r<1
$$

то при некотором $B_{q}>0$ имеет место неравенство

$$
m_{q}(r, B)(1-r)^{1 / p} \geqslant B_{q} \int_{r}^{1} \frac{n(t)}{t} d t, \quad 0<r<1
$$

əде $q \in[1,+\infty)$.

Теорема 3. Пусть $1<q \leqslant 2$ и нули произведения Бляике $B, B \in \mathscr{B}_{a}$, положительны. Тогда для ограниченности функиии $m_{q}(r, B)$ необходимо и достаточно, чтобы

$$
n(r)=O\left((1-r)^{-1 / q}\right), \quad r \rightarrow 1
$$

Для доказательства этих результатов нам необходимы следуюшие леммы.

Лемма 1. Пусть $B \in \mathscr{B}_{a}$. Тогда существует постоянная $A_{q}$ такая, что при $q \geqslant 2,0<r<1, \rho=r(1-\sqrt{1-r})^{1 / p}$ имеет место неравенство

$$
\begin{aligned}
(1-r)^{1 / p} m_{q}(r) \leqslant A_{q} \max \{ & (n(r)-n(\rho))(1-r), n(r)(1-r)^{1+1 /(4 p)}, \\
& \left.\int_{r}^{1}(1-t) d n(t),(1-r)^{1 / p} \int_{r}^{1} n(t) d t\right\} .
\end{aligned}
$$

ЛЕмма 2 [6]. Пусть $p>1$,

$$
\psi(r)=\sum_{k=1}^{\infty}\left(\frac{1-r^{k}}{k}\right)^{p}, \quad 0<r<1
$$

Тогда $\psi(r) \leqslant q(1-r)^{p-1} n p u 0<r<1$. 
ДоКАЗАТЕЛЬСТВО ЛЕМмы 1 . Пусть $c_{k}(r)$ - коэффициенты Фурьев разложении функции $\ln \left|B\left(r e^{i \theta}\right)\right|$ по системе $\left\{e^{i k \theta}\right\}, k \in \mathbb{Z}$. В силу неравенства Хаусдорфа-Юнга при $q \geqslant 2$ и равенства $c_{k}=\bar{c}_{-k}$ имеем

$$
m_{q}(r, B) \leqslant\left(\sum_{k=-\infty}^{+\infty}\left|c_{k}(r)\right|^{p}\right)^{1 / p} \leqslant\left|c_{0}\right|+2\left(\sum_{k=1}^{\infty}\left|c_{k}(r)\right|^{p}\right)^{1 / p} .
$$

Коэффициенты Фурье $c_{k}(r)$ можно выразить через $a_{n}$ следуюшим образом [1]:

$$
\begin{gathered}
c_{0}(r)=-\int_{r}^{1} \frac{n(t)}{t} d t \\
c_{k}(r)=\frac{1}{2 k} \sum_{n=1}^{\infty}\left(\bar{a}_{n}^{k}-a_{n}^{-k}\right) r^{k}+\frac{1}{2 k} \sum_{\left|a_{n}\right| \leqslant r}\left(\left(\frac{r}{a_{n}}\right)^{k}-\left(\frac{\bar{a}_{n}}{r}\right)^{k}\right) \\
=\frac{1}{2 k} \sum_{\left|a_{n}\right| \leqslant r}\left(\left(\bar{a}_{n} r\right)^{k}-\left(\frac{\bar{a}_{n}}{r}\right)^{k}\right)+\frac{r^{k}}{2 k} \sum_{\left|a_{n}\right|>r} a_{n}^{-k}\left(\left|a_{n}\right|^{2 k}-1\right) .
\end{gathered}
$$

Обозначим модуль последней суммы в (6) через $J_{k}$, а предпоследней через $I_{k}$. Тогда

$$
I_{k} \leqslant \frac{1-r^{2 k}}{2 k r^{k}} \int_{0}^{r} t^{k} d n(t) \text {. }
$$

При $0<\rho<r$ имеем

$$
I_{k} \leqslant \frac{1-r^{2 k}}{2 k r^{k}}\left(\int_{0}^{\rho} t^{k} d n(t)+\int_{\rho}^{r} t^{k} d n(t)\right) \leqslant \frac{1}{2 k}\left(1-r^{2 k}\right)\left(\left(\frac{\rho}{r}\right)^{k} n(\rho)+n(r)-n(\rho)\right) .
$$

Обозначим $t^{-1}=e^{x}$. Тогда

$$
\frac{t^{-k}-t^{k}}{t^{-1}-t}=\frac{\operatorname{sh} k x}{\operatorname{sh} x}
$$

Учитьвая, что функция $\operatorname{sh} k x / \operatorname{sh} x$ возрастающая по $x$, имеем

$$
\begin{aligned}
J_{k} & \leqslant \frac{r^{k}}{2 k} \int_{r}^{1} t^{-k}\left(1-t^{2 k}\right) d n(t)=\frac{r^{k}}{2 k} \int_{r}^{1} \frac{t^{-k}-t^{k}}{t^{-1}-t}\left(t^{-1}-t\right) d n(t) \\
& \leqslant \frac{r^{k}}{2 k} \frac{r^{-k}-r^{k}}{r^{-1}-r} \int_{r}^{1}\left(t^{-1}-t\right) d n(t)=\frac{r^{k}}{2 k} \frac{r^{-k}\left(1-r^{2 k}\right)}{r^{-1}\left(1-r^{2}\right)} \int_{r}^{1} \frac{1-t^{2}}{t} d n(t) .
\end{aligned}
$$

Вьполняя элементарные оценки, получаем

$$
J_{k} \leqslant 2 \frac{1-r^{k}}{k(1-r)} \int_{r}^{1}(1-t) d n(t)
$$

Используя $(7),(8)$ и неравенство Гёльдера, находим

$$
\begin{aligned}
\left(\sum_{k=1}^{\infty}\left|c_{k}\right|^{p}\right)^{1 / p} \leqslant & \left(\sum _ { k = 1 } ^ { \infty } \left(\frac{1-r^{2 k}}{2 k}(n(r)-n(\rho))+\frac{1-r^{2 k}}{2 k}\left(\frac{\rho}{r}\right)^{k} n(\rho)\right.\right. \\
& \left.\left.+2 \frac{1-r^{k}}{k} \frac{1}{1-r} \int_{r}^{1}(1-t) d n(t)\right)^{p}\right)^{1 / p} \\
\leqslant & \left(\sum_{k=1}^{\infty}\left(\frac{1-r^{2 k}}{2 k}(n(r)-n(\rho))\right)^{p}\right)^{1 / p}+\left(\sum_{k=1}^{\infty}\left(\frac{1-r^{2 k}}{2 k}\left(\frac{\rho}{r}\right)^{k}\right)^{p}\right)^{1 / p} \\
& +2\left(\sum_{k=1}^{\infty}\left(\frac{1-r^{k}}{k} \frac{1}{1-r} \int_{r}^{1}(1-t) d n(t)\right)^{p}\right)^{1 / p}
\end{aligned}
$$


В силу леммы 2 имеем

$$
\begin{aligned}
\left(\sum_{k=1}^{\infty}\left(\frac{1-r^{2 k}}{2 k}(n(r)-n(\rho))\right)^{p}\right)^{1 / p} & \leqslant\left(q\left(1-r^{2}\right)^{p-1}\right)^{1 / p}(n(r)-n(\rho)) \\
& \leqslant q^{1 / p} 2^{(p-1) / p}(1-r)^{1-1 / p}(n(r)-n(\rho)) .
\end{aligned}
$$

Полагая $\rho=r(1-\sqrt{1-r})^{1 / p}$ и применяя неравенство Коши и лемму 2 , получаем

$$
\begin{aligned}
& \left(\sum_{k=1}^{\infty}\left(\frac{1-r^{2 k}}{2 k}\left(\frac{\rho}{r}\right)^{k} n(\rho)\right)^{p}\right)^{1 / p}=\left(\sum_{k=1}^{\infty}\left(\frac{1-r^{2 k}}{2 k}(1-\sqrt{1-r})^{k / p} n(\rho)\right)^{p}\right)^{1 / p} \\
& \quad=n(\rho)\left(\sum_{k=1}^{\infty}\left(\frac{1-r^{2 k}}{2 k}\right)^{p}(1-\sqrt{1-r})^{k}\right)^{1 / p} \\
& \quad \leqslant n(\rho)\left(\sum_{k=1}^{\infty}\left(\frac{1-r^{2 k}}{2 k}\right)^{2 p}\right)^{1 /(2 p)}\left(\sum_{k=1}^{\infty}(1-\sqrt{1-r})^{2 k}\right)^{1 /(2 p)} \\
& \quad \leqslant n(\rho) q\left(1-r^{2}\right)^{(2 p-1) /(2 p)}\left(\frac{1}{1-(1-\sqrt{1-r})^{2}}\right)^{1 /(2 p)} .
\end{aligned}
$$

С помошью элементарных преобразований имеем

$$
\left(\sum_{k=1}^{\infty}\left(\frac{1-r^{2 k}}{2 k}\left(\frac{\rho}{r}\right)^{k} n(\rho)\right)^{p}\right)^{1 / p} \leqslant q 2^{1-1 /(2 p)} n(r)(1-r)^{1-3 /(4 p)} .
$$

Для оценки последней суммы в (9) снова воспользуемся леммой 2. Тогда

$$
\begin{aligned}
\left(\sum_{k=1}^{\infty}\left(\frac{1-r^{k}}{k} \frac{1}{1-r} \int_{r}^{1}(1-t) d n(t)\right)^{p}\right)^{1 / p} & \leqslant \frac{1}{1-r} \int_{r}^{1}(1-t) d n(t) q(1-r)^{1-1 / p} \\
& =q(1-r)^{-1 / p} \int_{r}^{1}(1-t) d n(t)
\end{aligned}
$$

Учитьвая, что

$$
\left|c_{0}(r, B)\right|=\int_{r}^{1} \frac{n(t)}{t} d t \leqslant \frac{1}{a} \int_{r}^{1} n(t) d t,
$$

и выбирая $A_{q}=4 \max \left\{q^{1 / p} 2^{1 / p}, q^{1-1 /(2 p)}, 2 q, 1 / a\right\}$, из (4) и (10)-(12) получаем (3).

ДоКАЗАТЕЛЬСТво ТЕОРЕМЫ 1. Используя очевидные неравенства

$$
\begin{gathered}
n(r)(1-r)<\int_{r}^{1} n(t) d t<\int_{r}^{1} \frac{n(t)}{t} d t \\
(n(r)-n(\rho))(1-r)<n(r)(1-r), \quad n(r)(1-r)^{1+1 /(4 p)}<n(r)(1-r) \\
(1-r)^{1 / p} \int_{r}^{1} n(t) d t<\int_{r}^{1} n(t) d t \\
\int_{r}^{1}(1-t) d n(t)=-n(r)(1-r)+\int_{r}^{1} n(t) d t<\int_{r}^{1} n(t) d t
\end{gathered}
$$


и лемму 1, получаем $(1)$ при $q \geqslant 2$.

Рассмотрим теперь случай $1 \leqslant q<2$. Обозначим $F(q)=m_{q}(r)$. Функция $\ln F(q)$ выпукла относительно $1 / q[7$, с. 236], т.е.

$$
\ln F(q) \leqslant(1-\theta) \ln F(\omega)+\theta \ln F(s)
$$

при $1 / q=(1-\theta) / \omega+\theta / s, 0 \leqslant \theta \leqslant 1$, или

$$
m_{q}(r) \leqslant m_{\omega}(r)^{1-\theta} m_{s}(r)^{\theta} .
$$

Положим $\omega=1, s=2$. Тогда $\theta=2 / p$ и

$$
m_{q}(r) \leqslant m_{1}(r)^{2 / q-1} m_{2}(r)^{2 / p} .
$$

Кроме того, в силу (5) и неравенства $\left|B\left(r e^{i \theta}\right)\right|<1$ имеем

$$
m_{1}(r)=\int_{0}^{2 \pi}|\ln | B\left(r e^{i \theta}\right)|| d \theta=-\int_{0}^{2 \pi} \ln \left|B\left(r e^{i \theta}\right)\right| d \theta=\int_{r}^{1} \frac{n(t)}{t} d t
$$

а в силу (1) при $q=2$ получаем

$$
m_{2}(r) \leqslant A_{2}(1-r)^{-1 / 2} \int_{r}^{1} \frac{n(t)}{t} d t .
$$

Тогда (14) можно переписать в виде

$$
m_{q}(r) \leqslant A_{2}^{2-2 / q}(1-r)^{1 / q-1} \int_{r}^{1} \frac{n(t)}{t} d t .
$$

Таким образом,

$$
(1-r)^{1 / p} m_{q}(r, B) \leqslant A_{q} \int_{r}^{1} \frac{n(t)}{t} d t
$$

для $q \in[1,+\infty), 0<r<1$, где

$$
A_{q}= \begin{cases}4 \max \left\{q^{1 / p} 2^{1 / q}, q 2^{1-1 /(2 p)}, 2 q, 1 / a\right\} & \text { при } q \geqslant 2, \\ A_{2}^{2 / p} & \text { при } 1 \leqslant q \leqslant 2,\end{cases}
$$

и теорема 1 доказана.

ДоКАЗАТЕЛЬСТво ТЕОРЕМЫ 2. Используя неравенство Хаусдорфа-Юнга при $1<$ $q \leqslant 2$, имеем

$$
\left(\frac{1}{2 \pi} \int_{0}^{2 \pi}\left(\ln \left|B\left(r e^{i \theta}\right)\right|\right)^{q} d \theta\right)^{1 / q} \geqslant\left|c_{0}(r)\right|+2\left(\sum_{k=1}^{\infty}\left|c_{k}(r)\right|^{p}\right)^{1 / p} .
$$

Оценим при $p \geqslant 2$ сумму в правой части. Имеем

$$
\begin{aligned}
\left|c_{k}(r)\right|^{p} & =\left|\left(\frac{1}{2 k}\right)^{p}\left(\left(r^{k}-r^{-k}\right) \sum_{a_{n} \leqslant r} a_{n}^{k}+r^{k} \sum_{a_{n} \geqslant r}\left(a_{n}^{k}-a_{n}^{-k}\right)\right)^{p}\right| \\
& \geqslant\left(\frac{1}{2 k}\right)^{p}\left(r^{k} \sum_{a_{n} \geqslant r}\left(a_{n}^{-k}-a_{n}^{k}\right)\right)^{p}=\left(\frac{1}{2 k}\right)^{p}\left(r^{k} \sum_{a_{n} \geqslant r} \frac{\left(1-a_{n}^{k}\right)\left(1+a_{n}^{k}\right)}{a_{n}^{k}}\right)^{p} \\
& =\left(r^{k} \sum_{a_{n} \geqslant r} \frac{\left(1-a_{n}\right)\left(1+a_{n}+\cdots+a_{n}^{k-1}\right)\left(1+a_{n}^{k}\right)}{2 k a_{n}^{k}}\right)^{p}
\end{aligned}
$$


Учитьвая, что $1+a_{n}+\cdots+a_{n}^{k-1} \geqslant k a_{n}^{k}$, получаем

$$
\left|c_{k}(r)\right|^{p} \geqslant \frac{1}{2^{p}} r^{p k}\left(\int_{r}^{1}(1-t) d n(t)\right)^{p} .
$$

Tак как $r<1$, имеет место

$$
\sum_{k=1}^{\infty} r^{p k}=\frac{1}{1-r^{p}}
$$

и

$$
\begin{aligned}
m_{q}(r, B) & \geqslant 2\left(\sum_{k=1}^{\infty}\left|c_{k}(r)\right|^{p}\right)^{1 / p}=\left(\frac{1}{1-r^{p}}\right)^{1 / p} \int_{r}^{1}(1-t) d n(t) \\
& \geqslant p^{-1 / p}(1-r)^{-1 / p} \int_{r}^{1}(1-t) d n(t)
\end{aligned}
$$

Интегрируя по частям и используя условие (2), получаем

$$
2\left(\sum_{k=1}^{\infty}\left|c_{k}(r)\right|^{p}\right)^{1 / p} \geqslant p^{-1 / p}(1-r)^{-1 / p}(1-\beta) \int_{r}^{1} n(t) d t
$$

Учитьвая, что

$$
\int_{r}^{1} n(t) d t \geqslant a \int_{r}^{1} \frac{n(t)}{t} d t
$$

для $1 \leqslant q \leqslant 2$ имеем

$m_{q}(r) \geqslant 2(1-\beta) p^{-1 / p}(1-r)^{-1 / p} \int_{r}^{1} n(t) d t \geqslant 2 a(1-\beta) p^{-1 / p}(1-r)^{-1 / p} \int_{r}^{1} \frac{n(t)}{t} d t$.

Обозначим $B_{q}=2 a(1-\beta) p^{-1 / p}$. Тогда

$$
m_{q}(r, B)(1-r)^{1 / p} \geqslant B_{q} \int_{r}^{1} \frac{n(t)}{t} d t .
$$

Чтобы доказать теорему для $q \geqslant 2$, воспользуемся приемом из доказательства теоремы 1 при $1<q \leqslant 2$. Перепишем (13) в виде

$$
m_{s} \leqslant m_{\omega}^{1-\theta} m_{q}^{\theta}
$$

при $1 / s=(1-\theta) / \omega+\theta / q$, где $0 \leqslant \theta \leqslant 1$.

Положим $s=2, \omega=1$. Тогда $\theta=q /(2(q-1))$. Как было доказано,

$$
m_{2}(r, B) \geqslant B_{2} \int_{r}^{1} \frac{n(t)}{t} d t, \quad m_{1}(r, B)=\int_{r}^{1} \frac{n(t)}{t} d t .
$$

Тем самьм, согласно (16) получим

$$
B_{2}(1-r)^{-1 / 2} \int_{r}^{1} \frac{n(t)}{t} d t \leqslant\left(\int_{r}^{1} \frac{n(t)}{t} d t\right)^{1-\theta}\left|m_{q}(r)\right|^{\theta}
$$


откуда

$$
B_{2}^{1 / \theta}(1-r)^{(1-q) / q} \int_{r}^{1} \frac{n(t)}{t} d t \leqslant m_{q}(r) .
$$

Таким образом,

$$
m_{q}(r, B)(1-r)^{1 / p} \geqslant B_{q} \int_{r}^{1} \frac{n(t)}{t} d t
$$

где

$$
B_{q}= \begin{cases}2(1-\beta) p^{-1 / p} & \text { при } 1 \leqslant q \leqslant 2, \\ B_{2}^{2 / p} & \text { при } q \geqslant 2 .\end{cases}
$$

Тем самым, теорема 2 доказана.

Покажем теперь, что условие (2) существенно. Рассмотрим функцию

$$
n(r)=\left[\ln +\ln \frac{1}{1-r}\right]
$$

Покажем, что

$$
(1-r)^{1 / p} m_{q}(r)=o\left(\int_{r}^{1} \frac{n(t)}{t} d t\right), \quad r \rightarrow 1 .
$$

При $\rho=r(1-\sqrt{1-r})^{1 / p}$ имеем

$$
\begin{aligned}
n(\rho) & \geqslant n(r(1-\sqrt{1-r}))=\left[\ln +\ln \frac{1}{\sqrt{1-r}(r+\sqrt{1-r})}\right] \\
& =\ln \ln \frac{1}{1-r}+O(1), \quad r \rightarrow 1 .
\end{aligned}
$$

Следовательно, $n(r)-n(\rho)=O(1), r \rightarrow 1$. Таким образом,

$$
(n(r)-n(\rho))(1-r)=O(1-r), \quad r \rightarrow 1 .
$$

Очевидно, что

$$
\begin{gathered}
n(r)(1-r)^{1+1 /(4 p)} \leqslant\left(\ln ^{+} \ln \frac{1}{1-r}\right)\left(1-r^{1 /(4 p)}\right)(1-r)=o(1-r), \quad r \rightarrow 1, \\
\int_{r}^{1}(1-t) d n(t) \leqslant \int_{r}^{1} \frac{1}{-\ln (1-t)} d t=O(1-r), \quad r \rightarrow 1, \\
(1-r)^{1 / p} \int_{r}^{1} n(t) d t=o\left(\int_{r}^{1} n(t) d t\right), \quad r \rightarrow 1 .
\end{gathered}
$$

Поскольку

$$
1-r \leqslant \frac{1}{n(r)} \int_{r}^{1} n(t) d t=o\left(\int_{r}^{1} n(t) d t\right), \quad r \rightarrow 1,
$$

используя лемму $1,(18)$ и (19), получаем (17). 
ДоКАЗАТЕЛЬСТво СЛЕДСТВИЯ. Из неравенства (1) следует

$$
(1-r)^{1 / p_{m}} m_{q}(r, B) \leqslant \frac{A_{q}}{a} \int_{r}^{1} n(t) d t .
$$

При $n(r)=O\left((1-r)^{-\alpha}\right)$ имеем

$$
\frac{A_{q}}{a} \int_{r}^{1} n(t) d t=O\left(\int_{r}^{1}(1-t)^{-\alpha} d t\right)=O\left((1-r)^{-\alpha+1}\right), \quad r \rightarrow 1 .
$$

Следовательно,

$$
m_{q}(r, B)=O\left((1-r)^{-\alpha+1-1 / p}\right)=O\left((1-r)^{1 / q-\alpha}\right), \quad r \rightarrow 1 .
$$

ДОКАЗАТЕЛЬСТВО ТЕОРЕМЫ 3. Необходимость. Пусть функция $m_{q}(r, B)$ ограничена. Используем полученный результат (15) в следующем виде:

$$
(1-r)^{1 / p} m_{q}(r) \geqslant B \int_{r}^{1}(1-t) d n(t)
$$

где $B=$ const.

Согласно условию Бляшке

$$
n(r)=-\int_{0}^{r} \frac{d \int_{t}^{1}(1-\tau) d n(\tau)}{1-t} \leqslant \int_{0}^{1}(1-\tau) d n(\tau)+\int_{0}^{r} \frac{\int_{t}^{1}(1-\tau) d n(\tau)}{(1-t)^{2}} d t
$$

откуда

$$
\begin{aligned}
n(r) & \leqslant O(1)+\int_{0}^{r} \frac{\int_{t}^{1}(1-\tau) d n(\tau)}{(1-t)^{2}} d t=O(1)+\int_{0}^{r} \frac{(1-t)^{1 / p_{m}}(t)}{(1-t)^{2}} d t \\
& =O\left((1-r)^{-1 / q}\right), \quad r \rightarrow 1
\end{aligned}
$$

Достаточность получается из следствия при $\alpha=1 / q$. Заметим, что в этом случае $1<q<\infty$ и из условия Бляшке и соотношения $(8)$ всегда следует $m_{1}(r, B)=o(1)$, $r \rightarrow 1$.

\section{СПИСОК ЦИТИРОВАННОЙ ЛИТЕРАТУРЫ}

[1] McLane G. R., Rubel L. A. On the growth of Blaschke products // Canad. J. Math. 1969. V. 21. P. 595-601.

[2] Linden C. N. Integral means and zero distributions of Blaschke products // Canad. J. Math. 1972. V. 24. P. $755-760$.

[3] Linden C. N. Integral logarithmic means for regular functions // Pacific J. Math. 1989. V. 138. P. 119-127.

[4] Linden C. N. The characterization of orders for regular functions // Math. Proc. Cambridge Philos. Soc. 1992. V. 11. P. 299-307.

[5] Кондратюк А. А. Ряды Фурье и мероморфные функции. Львов: Выща школа, 1988.

[6] Кондратюк А.А., Тарасюк С.И. Сравнение лебеговых средних и неванлинновской характеристики субгармонических функций // Матем. студии. Тр. Львовского матем. об-ва. 1991. Вып. 1. С. 74-80.

[7] Бурбаки Н. Меры. Интегрирование мер. М.: Наука, 1987. 